\section{Dermatoporosis en estadio IV: a propósito de un caso}

\section{Stage IV dermatoporosis: a case report}

\author{
Cristina Bandera García ${ }^{1 * *}$ \\ Antonio Enrique Aragonés Domínguez² \\ Diego Lozano Noriega ${ }^{3}$ \\ Leovigildo Ginel Mendoza ${ }^{4}$ \\ Rafael Poyato Ramos²
}

\author{
1. Residente de Enfermería Familiar y Comunitaria. Centro de Salud Ciudad Jardín. \\ Málaga. España. \\ 2. Enfermero de familia. Centro de Salud Ciudad Jardín. Málaga. España. \\ 3. Enfermero especialista en Enfermería Familiar y Comunitaria. Centro de Salud \\ Canterac. Valladolid. España. \\ 4. Médico de familia. Centro de Salud Ciudad Jardín. Málaga. España. \\ *Autor para correspondencia. \\ Correo electrónico: cristinabangar@gmail.com (Cristina Bandera García)
}

Recibido el 7 de septiembre de 2018; aceptado el 18 de octubre de 2018

\section{RESUMEN}

Paciente de 79 años, remitida a consulta de enfermería para valoración de lesión postraumática en miembro inferior derecho de una semana de evolución, tratada con vendaje elástico y heparina de bajo peso molecular. Objetivo: Conocer el rol de la enfermería en la prevención y tratamiento de la dermatoporosis, a propósito de un caso clínico. Metodología: Caso clínico con planteamiento metodológico, con consentimiento informado de la paciente y fotografías del caso. Resultado: La cicatrización se consiguió en un periodo de 65 días, con una correcta preparación del lecho de la herida para el posterior éxito del injerto cutáneo, controlando el edema, exudado y dolor durante las curas, realizadas cada 2-3 días de acuerdo con la efectividad del tratamiento. Discusión: La dermatoporosis exige un mayor esfuerzo en prevención primaria y secundaria desde Atención Primaria, sobre todo a partir de la detección de las manifestaciones clínicas y complicaciones iniciales derivadas de la misma.

PALABRAS CLAVE: Dermatoporosis, desbridamiento, injerto cutáneo.

\section{ABSTRACT}

A 79-year-old patient referred to a nursing consultation to evaluate a traumatic lesion in the right lower limb for one week, treated with elastic bandage and low molecular weight heparin. Objective: Knowing the role of nursing in the prevention and treatment of dermatoporosis, in relation to a clinical case. Methodology: Clinical case with methodological approach, with informed consent of the patient and photographs. Result: The healing was achieved in a period of 65 days, with a correct preparation of the wound bed for the subsequent success of the skin graft, treating the edema, exudate and pain during the cures, made every 2-3 days in accordance with the effectiveness of the treatment. Discussion: Dermatoporosis requires a greater effort in primary and secondary prevention from primary care, especially from the detection of clinical manifestations and initial complications derived from it.

KEYWORDS: Dermatoporosis, debridement, skin graft.

\section{- INTRODUCCIÓN}

La piel es el mayor órgano de nuestro cuerpo, del que destaca su función barrera/protectora contra las agresiones externas e internas.

Con el paso de los años, las funciones metabólicas y fisiológicas de la piel se van perdiendo, de modo que el envejecimiento cutáneo se traduce en sequedad de la piel, pérdida de elasticidad, disminución del espesor epidérmico y dérmico y fragilidad cutánea, esta última directamente relacionada con las erosiones dérmicas, similares a la etiología de úlceras por cizallamiento ${ }^{1}$.

Directamente relacionado con estas úlceras, el término dermatoporosis fue acuñado en 2007 por Kaya y Saurat para dar nombre a la fragilidad cutánea crónica derivada del envejecimiento de la piel. Con ello pretendían que la dermatoporosis transmitiera la vulnerabilidad en la piel, haciendo el símil con la osteoporosis en los huesos, trasladando así la necesidad de su prevención. La dermatoporosis, cuyos primeros signos y síntomas aparecen a partir de los 60 años y se manifiestan por completo entre los 70 y los 90 años, define aquellas manifestaciones clínicas y las complicaciones derivadas de la insuficiencia cutánea crónica o síndrome de fragilidad cutánea, tales como la púrpura solar, seudoescaras blanquecinas, laceraciones en la piel y curación retrasada.

Estos mismos autores describieron cuatro estadios, descritos en la tabla $1^{2,3}$.

\section{- HISTORIA CLÍNICA}

Paciente de 79 años que presenta, como antecedentes personales de interés, obesidad de tipo II, hipertensión y diabetes mellitus tipo 2 .

En cuanto al nivel de autonomía para la realización de las actividades básicas de la vida diaria, evaluadas con el Índice de Barthel ${ }^{5}$, presenta una dependencia leve.

A nivel sociofamiliar, valorado con la Escala de valoración socio-fami$\operatorname{liar}^{6}$, la paciente cuenta con un buen núcleo familiar (marido y dos hi- 
Tabla 1. Características clínicas de la dermatoporosis

\begin{tabular}{|c|c|c|c|c|}
\hline Características clínicas & Estadio I & Estadio II & Estadio III & Estadio IV \\
\hline Atrofia cutánea: arrugas muy delgadas y numerosas & + & + & + & + \\
\hline Púrpura senil: sangrado espontáneo repetitivo en la dermis sin anomalías de la coagulación & + & + & + & + \\
\hline Laceración de la piel: expresión de la fragilidad de la piel después de un trauma menor & + & + & + & + \\
\hline Cicatrices estrelladas: laceraciones espontáneas de la piel con apariencia estelar & - & $+(<10)$ & $++(\geq 10)$ & $++(\geq 10)$ \\
\hline Hematoma de disección de la piel: hemorragia subcutánea, que lleva a la isquemia y a la necrosis de la piel & - & - & - & + \\
\hline
\end{tabular}

Recuperado de: Domínguez et al4

jos) y social, sin riesgo social. La Escala de Zarit ${ }^{7}$ indica que su cuidador (su marido) no tiene sobrecarga en su rol.

A continuación, se presentan los datos sobre las patologías crónicas que padece la paciente:

- IMC: $38 \mathrm{~kg} / \mathrm{m}^{2}$.

- Tensión arterial: 130/70 mmHg.

- Frecuencia cardíaca: $68 \mathrm{lpm}$.

- Hemoglobina glicosilada: 7,7\%.

- Cribado anual de neuropatía diabética: sensibilidad vibratoria a diapasón, normal. Sensibilidad barestésica normal. Pulso pedio y tibial posterior disminuidos.

\section{- VALORACIÓN INICIAL DEL CASO CLÍNICO}

La paciente es remitida a consulta de enfermería para valoración de lesión postraumática en miembro inferior derecho. A la retirada del vendaje elástico, aplicado hace 7 días, se observa gran hematoma subcutáneo (lecho necrótico seco), de tamaño aproximado $20 \times 8 \mathrm{~cm}$ (fig. 1), que abarca la región gemelar desde el hueco poplíteo hasta la zona supramaleolar de dicha extremidad. No presenta exudado ni olor. La piel perilesional presenta buen estado.

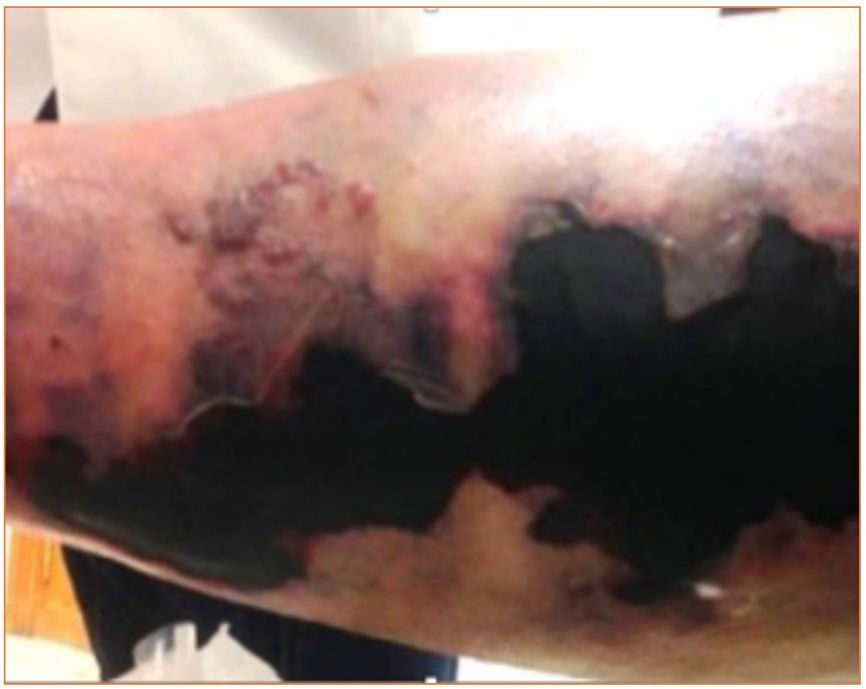

Figura 1. Primer día de cura de úlcera postraumática en región gemelar, miembro inferior derecho.
La lesión, como se observa en la figura 1, es compatible con las características clínicas de la dermatoporosis de estadio IV.

\section{- DIAGNÓSTICO}

Diagnósticos de enfermería: deterioro de la integridad cutánea (00046).

Diagnósticos según CIE-10: úlcera crónica no debida a presión de pantorrilla derecha, con exposición de la capa adiposa (L97.212).

\section{- PLAN DE ACTUACIÓN Y EVOLUCIÓN}

Se planificaron los objetivos a conseguir de acuerdo con criterios de resultados NOC y se adecuaron las intervenciones NIC necesarias para ello, como se muestra en la tabla 2.

Las curas se realizaron en ambiente húmedo, siguiendo para su abordaje el concepto TIME, y su evolución fue lenta debido a la gran cantidad de tejido necrótico seco del lecho de la lesión, resultado del hematoma profundo disecante. El tejido no viable se eliminó mediante desbridamiento autolítico, enzimático y cortante, usando para ello hidrogel, colagenasa y pinzas y bisturí, respectivamente. Para el control de la infección se utilizaron apósitos con plata y el exudado se gestionó con

Tabla 2. Indicadores de resultados asociados al criterio de resultado NOC

\begin{tabular}{|c|c|c|c|c|c|}
\hline $\begin{array}{l}\text { Curación de la herida por } \\
\text { segunda intención (1103). } \\
\text { Indicadores }\end{array}$ & 1 & 2 & 3 & 4 & 5 \\
\hline Necrosis 110312 & $A, B$ & & C & & $\mathrm{D}$ \\
\hline Piel macerada 110311 & & & B & & D \\
\hline $\begin{array}{l}\text { Secreción serosanguinolenta } \\
110306\end{array}$ & $A, B$ & C & & & $\mathrm{D}$ \\
\hline Excavación 110315 & B & & C & & $A, D$ \\
\hline \multicolumn{6}{|c|}{ 1: Extenso. 2: Sustancial. 3: Moderado. 4: Escaso. 5: Ninguno. } \\
\hline Disminución de tamaño 110321 & A & $B, C$ & & & $\mathrm{D}$ \\
\hline Formación de cicatriz 110320 & & $B, C$ & & & D \\
\hline
\end{tabular}

A: Valoración inicial. B: Valoración a los 10 días. C: Valoración a los 17 días. D: Valoración a los 57 días. 


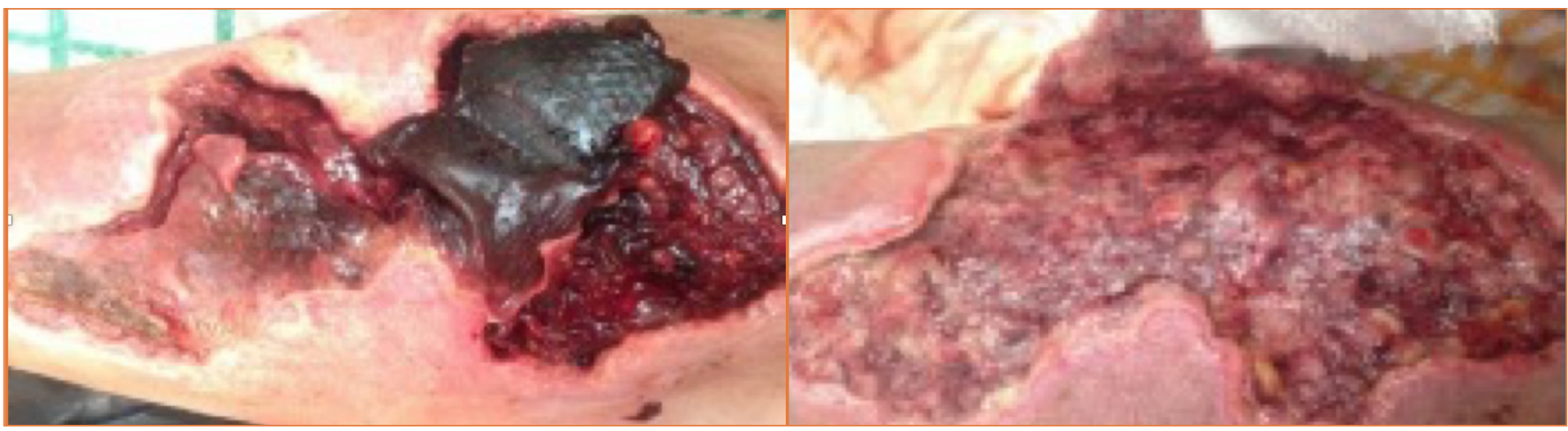

Figura 2. Décimo y decimoséptimo día de cura de úlcera postraumática en región gemelar, miembro inferior derecho.

hidrofibra de hidrocoloide/espuma de poliuretano, según las variaciones a lo largo del proceso. Para la protección de bordes se utilizó pasta Lassar.

La pauta de curas se estableció en el domicilio de la paciente (debido a incapacidad funcional y condiciones de acceso al domicilio), cada 2 días durante las dos primeras semanas. Se consiguió eliminar gran parte del tejido necrótico seco, aumentando considerablemente el exudado durante la primera semana de curas. A partir de los 10 días de tratamiento, se consiguió reducir la cantidad de exudado de la herida y el lecho de la herida se presentaba más limpio (fig. 2).

Debido a la previsible dificultad en la contracción de los bordes de la herida, teniendo en cuenta la extensión de la lesión y el colgajo en la parte interna de esta, además de los factores no facilitadores de la cicatrización propios de la paciente (anemia, leve desnutrición, obesidad y diabetes mellitus tipo 2), se comenta la evolución con su médico de familia, de forma que se deriva al servicio de cirugía plástica para valoración de injerto cutáneo.

El día 20 de evolución de la lesión, la paciente recibe un autoinjerto de espesor parcial, tomando como zona dadora la epidermis y dermis superficial del muslo derecho. La paciente recibe el alta hospitalaria el día 38 de evolución de la lesión, continuando las curas en domicilio por nuestra parte, tanto de la zona dadora como de la zona injertada, mediante limpieza con suero fisiológico, desinfección con povidona yodada y cobertura con gasa vaselinada y vendaje. En alguna cura realizada, debido a un leve crecimiento de fibrina en zonas delimitadas del injerto, también hubo que recurrir al desbridamiento cortante. Con los cuidados indicados, fundamentados en la evidencia científica disponible, el injerto queda totalmente prendido y cicatrizado a los 57 días desde que se produjo la lesión (fig. 3).

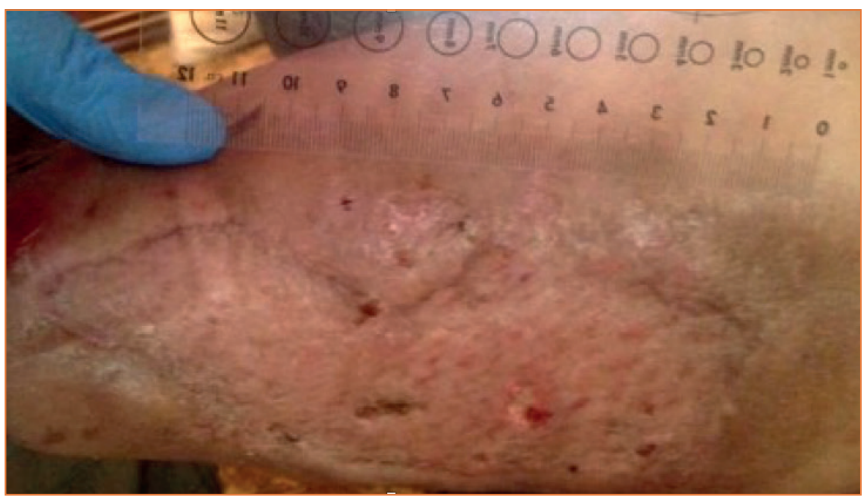

Figura 3. Injerto prendido y cicatrizado el día 57 desde el inicio de las curas.

\section{- DISCUSIÓN}

Los profesionales de enfermería, en la atención a los ancianos, han de ser sensibles ante un problema como la dermatoporosis, la cual es un factor predisponente para el desarrollo de desgarros cutáneos y para el retraso en la cicatrización de la lesión ${ }^{3}$.

En el caso que se presenta, las características de la dermatoporosis fueron identificadas en la región gemelar del miembro inferior derecho, a modo de hematoma de disección de la piel.

A nivel de tratamiento, se destaca la importancia de indicar una pauta de curas acorde con la evolución y valoración del hematoma. En este contexto, la utilización del desbridamiento está indicada con el fin de eliminar el tejido necrótico a la mayor brevedad posible, bien sea con métodos enzimáticos (enzimas proteolíticas), físicos (pinzas y bisturí) o autolíticos (cura en ambiente húmedo) ${ }^{8}$.

Asimismo, el manejo del hematoma de disección de la piel en las personas mayores presenta muchos desafíos debido a los cambios que se producen derivados del envejecimiento y de la excesiva exposición al sol, la existencia de comorbilidades, especialmente enfermedad vascular, la polimedicación, incluido el uso de anticoagulantes y fármacos que afectan a la cicatrización? .

Además del desbridamiento y cura en ambiente húmedo utilizados en este caso clínico, otros autores indican que para evacuar este tipo de hematomas existen dos técnicas?

1. Aspiración con aguja. Aunque se ha usado ampliamente, este método ya no está recomendado por muchas fuentes debido a la posibilidad de reacumulación del hematoma.

2. Incisión y drenaje. Debe hacerse en la periferia del hematoma, donde la piel muestra una apariencia normal, y paralelamente a las líneas de tensión.

En el caso que se trata, no fue posible la aplicación de ninguna de estas dos técnicas, debido al tiempo de evolución desde el inicio de la lesión y la llegada a nuestra consulta. A la primera retirada del vendaje, una semana después de producirse la lesión, nos encontramos con una placa necrótica seca, producto del hematoma profundo disecante, que ya estaba completamente coagulado y se albergaba debajo de la placa.

\section{- CONCLUSIONES}

La dermatoporosis es una patología de reciente denominación, la cual se debe dar a conocer entre los profesionales sanitarios. La instrucción en su fisiopatología, etiología y tratamiento es crucial para la prevención 
de complicaciones, como la aparición de desgarros cutáneos o retraso en la cicatrización, siendo la más grave el hematoma profundo disecante con necrosis.

El progresivo envejecimiento de la población, traducido en un aumento de las personas ancianas, propicia que la incidencia de esta enfermedad sea cada vez mayor entre los pacientes que acuden a consulta de enfermería en Atención Primaria.
Todo lo anterior, asociado además a los altos costes directos e indirectos que conlleva, deriva en la necesidad de realizar más investigaciones que pongan el énfasis en la prevención y reducción de sus secuelas

\section{Conflicto de intereses}

Los autores declaran no tener ningún conflicto de intereses relacionado con este caso clínico.

\section{- BIBLIOGRAFÍA}

1. Palomar F, Fornes B, Arantón L, Rumbo JM. Envejecimiento cutáneo dermatoporosis. Enferm Dermatol [Internet]. 2013;7(18):8-13. Disponible en: https://dialnet.unirioja.es/servlet/articulo?codigo=4529974

2. Dyer JM, Miller RA. Chronic skin fragility of aging: current concepts in the pathogenesis, recognition, and management of Dermatoporosis. J Clin Aesthet Dermatol. [Internet]. 2018;11(1):138. Disponible en: https://www.ncbi.nlm.nih.gov/pmc/articles/ PMC5788262/

3. Vanzi V, Toma E. Recognising and managing age-related dermatoporosis and skin tears. Nurs Older People [Internet]. 2018:30(3):26-3 . Disponible en: https://pubmed.ncbi.nlm.nih.gov/29569862-recognising-and-managing-age-related-dermatoporosis-and-skin-tears/?from_term=Recognising+and+managing+age-related\&from_pos=2
4. Domínguez ML, Di Martino B, Rodríguez M, Knopfelmacher O, Argüello L. Dermatoporosis, an emerging disease: case report. Our Dermatol Online [Internet]. 2016:7(2):191-4. Disponible en: http:// www.odermatol.com/issue-in-html/2016-2-16/

5. Cid J, Damián J. Valoración de la discapacidad física: el índice de Barthel. Rev Esp Salud Pública [Internet]. 1997;71:127-37. Disponible en: http://scielo.isciii.es/pdf/resp/v71n2/barthel.pdf

6. Junta de Andalucía. Escala de valoración socio-familiar. Cuestionarios, test e índices de valoración enfermera en formato para uso clínico. Servicio Andaluz de Salud, Consejería de Salud. Disponible en: http://www.sspa.juntadeandalucia.es/servicioandaluzdesalud/contenidos/publicaciones/datos/95/pdf/cuestionario_completo_sas.pdf
7. Junta de Andalucía. Cuestionario Zarit. Cuestionarios, test e índices de valoración enfermera en formato para uso clínico. Servicio Andaluz de Salud, Consejería de Salud. Disponible en: http://www. sspa.juntadeandalucia.es/servicioandaluzdesalud/contenidos/publicaciones/datos/95/pdf/cuestionario_completo_sas.pdf

8. Asociación Española de Enfermería Vascular y Heridas. Guía de práctica clínica: Consenso sobre úlceras vasculares y pie diabético. 3. a ed. Madrid: AEEVH, 2017. Disponible en: http://www.aeev.net/ pdf/Guia-de-Practica-Clinica-web.pdf

9. Pagan M, Hunter J. Lower leg haematomas: Potential for complications in older people. Wound Practice and Research [Internet]. 2011 [citado 21 Dic 2017];19(1):21-8. Disponible en: https://www.nzwcs. org.nz/images/publications/Pagan-Hunter_WPR_19-01.pdf 